\title{
Community-acquired versus hospital-acquired acute kidney injury in patients with acute exacerbation of COPD requiring hospitalization in China
}

\author{
Chang-chun $\mathrm{Cao}^{1, *}$ \\ Da-wei Chen ${ }^{2, *}$ \\ Jing $\mathrm{Li}^{2}$ \\ Meng-qing $\mathrm{Ma}^{2}$ \\ Yu-bao Chen ${ }^{3}$ \\ Yi-zhi $\mathrm{Cao}^{4}$ \\ Xi Hua ${ }^{2}$ \\ Wei Shao' \\ Xin Wan ${ }^{2}$
}

'Department of Nephrology, Sir Run Run Hospital, Nanjing Medical University, Nanjing, Jiangsu, China; ${ }^{2}$ Department of Nephrology, Nanjing First Hospital, Nanjing Medical University, Nanjing, Jiangsu, China; ${ }^{3}$ Department of Respiratory Medicine, Nanjing First Hospital, Nanjing Medical University, Nanjing, Jiangsu, China; ${ }^{4}$ The First Clinical Medical College, Nanjing Medical University, Nanjing, Jiangsu, China

*These authors contributed equally to this work
This article was published in the following Dove Press journal: International Journal of COPD

Purpose: Previous studies have described the incidence, risk factors, and outcomes for patients with acute exacerbations of COPD (AECOPD) developing acute kidney injury (AKI). However, little is known about the differences between community-acquired AKI (CA-AKI) and hospitalacquired AKI (HA-AKI) in patients with AECOPD. Thus, in this study, we compared prevalence, risk factors, and outcomes for these patients with CA-AKI and HA-AKI.

Patients and methods: This study was conducted from January 2014 to January 2017, and data from adult inpatients with AECOPD were analyzed retrospectively. A total of 1,768 patients were included, 280 patients were identified with CA-AKI and 97 patients were with HA-AKI.

Results: Prevalence of CA-AKI was $15.8 \%$ and that of HA-AKI was $5.5 \%$, giving an overall AKI prevalence of $21.3 \%$. Patients with CA-AKI had a higher prevalence of chronic kidney disease (CKD) and lower prevalence of chronic cor pulmonale than patients with HA-AKI. Risk factors for developing HA-AKI and CA-AKI were similar, such as being elderly, requirement for mechanical ventilation, and a history of coronary artery disease and CKD. Patients with HAAKI were more likely to have stage $3 \mathrm{AKI}$ and worse short-term outcomes. In comparison with patients with CA-AKI, those with HA-AKI were more likely to require non-invasive mechanical ventilation $(31.3 \%$ versus $16.8 \% ; P=0.003)$ and had a longer duration of mechanical ventilation (11 days versus 8 days; $P=0.020$ ), longer hospitalization (14 days versus 12 days; $P=0.038$ ), and higher inpatient mortality $(32.0 \%$ versus $13.2 \% ; P<0.001)$. Patients with HA-AKI had worse (multivariate-adjusted) inpatient survival than those with CA-AKI (hazard ratio, 1.7 [95\% confidence interval, $1.03-2.81 ; P=0.038$ ] for the HA-AKI group).

Conclusion: AKI was common in patients with AECOPD requiring hospitalization. CA-AKI was more common than HA-AKI but otherwise demonstrated similar demographics and risk factors. Nevertheless, patients with HA-AKI had worse short-term outcomes.

Keywords: acute exacerbation of COPD, community-acquired acute kidney injury, hospitalacquired acute kidney injury, short-term outcomes

\section{Introduction}

COPD affects $9 \%-10 \%$ of people aged $>40$ years worldwide. ${ }^{1}$ Disease exacerbations have a profound effect on patients with COPD, resulting in poor health and high mortality. ${ }^{2,3}$ Acute kidney injury (AKI) can be a severe complication of COPD. AKI is a multifaceted syndrome that occurs in different settings.

Fabbian et $\mathrm{al}^{4}$ reported that AKI represents a very important predictive factor of in-hospital mortality in patients admitted because of COPD exacerbations. Barakat
Correspondence: Xin Wan Department of Nephrology, Nanjing First Hospital, Nanjing Medical University, 68 Changle Road, Nanjing, Jiangsu 210006, China

Email wanxin@njmu.edu.cn 
and coworkers ${ }^{5}$ stated that the incidence rates of $\mathrm{AKI}$ in the COPD cohort and patients with AECOPD were 128/100,000 person-years and $1.9 \%$, respectively. Although they described the incidence, risk factors, and outcomes for patients with AECOPD developing AKI, less attention was focused on AKI that developed in the community and how this may differ from hospital-acquired (HA)-AKI. ${ }^{5}$

In this study, we compared the incidence, risk factors, and short-term outcomes of patients admitted to the hospital with community-acquired (CA)-AKI with those with HA-AKI.

\section{Patients and methods}

\section{Patient selection}

The study protocol was approved by the Regional Human Research Ethics Committee of Nanjing First Hospital (Nanjing, China). Individual patient consent was waived on condition that all patient data were de-identified before evaluation because this study was a retrospective analysis. This study was conducted at Nanjing First Hospital from January 2014 to January 2017. The diagnostic criteria for AECOPD were as follows: 1) a history of COPD (the patient had been clearly diagnosed with COPD, with typical clinical COPD presentations, and the pulmonary functions); 2) a change in the patient's baseline dyspnea, cough, and/or sputum that was beyond normal day-to-day variations, was acute in onset, and could warrant a change in regular medication in a patient with underlying COPD. ${ }^{6}$ The inclusion criterion was COPD exacerbation necessitating hospitalization. Exclusion criteria were as follows: patients without full medical records, with a urinary-tract infection, with a history of chronic kidney disease (CKD) stage 5, and undergoing dialysis before hospital admission (Figure 1).

\section{Definitions of CA-AKI and HA-AKI}

AKI was defined as a serum creatinine ( $\mathrm{SCr}$ ) change that met the 2012 Kidney Disease Improving Global Outcomes criteria: an increase in the SCr level by $\geq 0.3 \mathrm{mg} / \mathrm{dL}$ within $48 \mathrm{~h}$ or $\geq 1.5$-fold from the baseline within 7 days. ${ }^{7}$ Any patient admitted to the hospital with AKI (CA-AKI) or who developed AKI during the hospital stay (HA-AKI) was identified and included in the analysis. Patients admitted to hospital with AKI apparent based on the first SCr measured within $24 \mathrm{~h}$ of hospital admission were denoted as having CA-AKI. In contrast, patients were identified as having HA-AKI, if AKI was not apparent upon hospital admission, but AKI developed during hospitalization. The baseline level of SCr was defined as the lowest one during hospitalization or during the preceding 12 months.

\section{Data collection}

The following data were collected from the medical records: sex, age, preexisting medical conditions (chronic cor pulmonale, pulmonary arterial hypertension, atrial fibrillation, hypertension, diabetes mellitus, coronary artery disease,

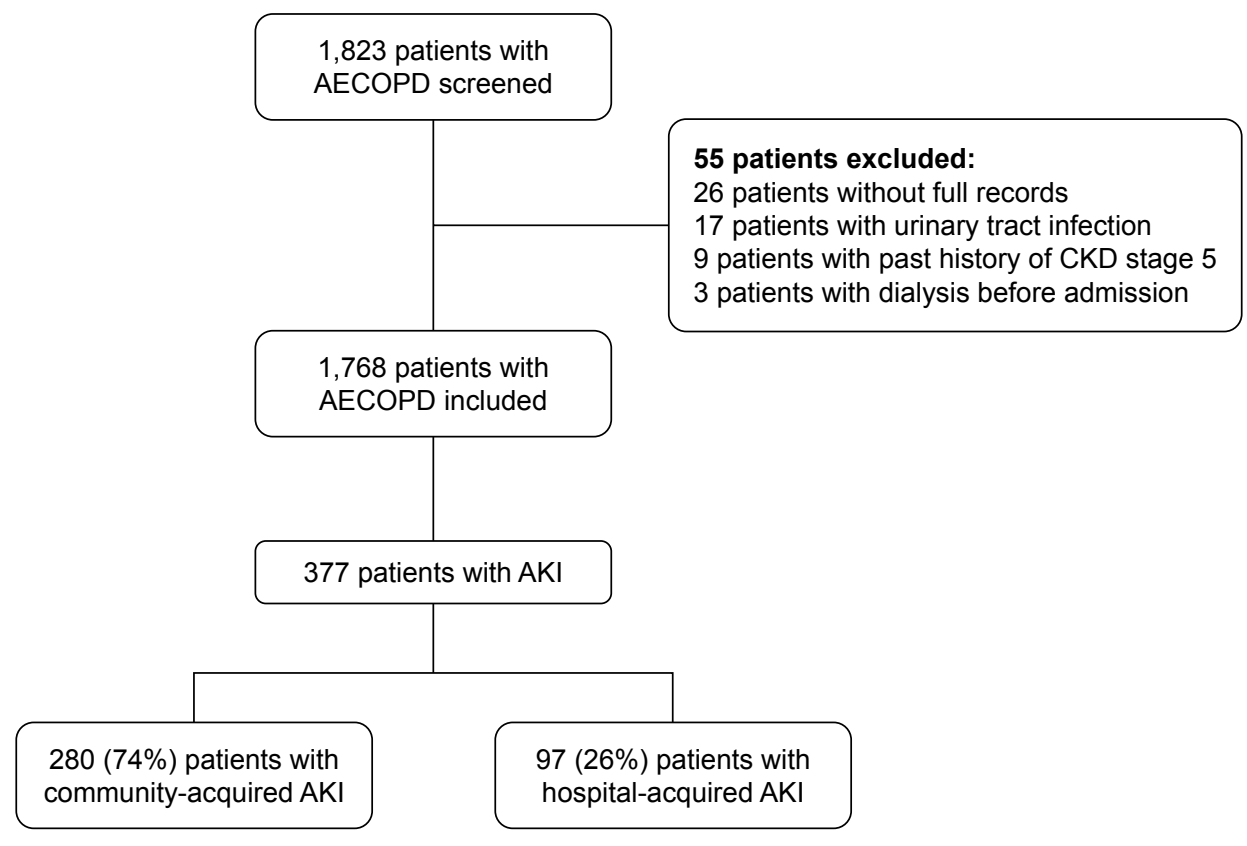

Figure I Flowchart for patient selection.

Abbreviations: AECOPD, acute exacerbations of COPD; AKI, acute kidney injury; CKD, chronic kidney disease. 
CKD, chronic liver disease, anemia, cerebrovascular disease, or cancer), complications (acute respiratory failure [ARF] and hypercapnic encephalopathy), requirement for mechanical ventilation (non-invasive mechanical ventilation, invasive mechanical ventilation, and duration of mechanical ventilation), requirement for renal replacement therapy (RRT), intensive care unit (ICU) admission, length of ICU stay, and duration of hospitalization. In-hospital outcomes such as the requirement for mechanical ventilation (non-invasive mechanical ventilation, invasive mechanical ventilation, and duration of mechanical ventilation), RRT and ICU admission, length of ICU stay, duration of hospitalization, and inpatient mortality were also documented.

\section{Data analyses}

Data were analyzed using SPSS v22.0 (IBM Corporation, Armonk, NY, USA). Continuous variables are presented as mean $\pm \mathrm{SD}$ or median values (25th-75th percentile) as appropriate. Categorical variables are shown as percentages. An unpaired Student's $t$-test was used to compare mean values between two groups. The Mann-Whitney $U$-test was employed to compare median values. Comparison between two groups for categorical variables was made using the chi-squared test and the Fisher's exact test where appropriate.

Univariate binary logistic regression analysis was done to evaluate the potential risk factors associated with $\mathrm{CA}-\mathrm{AKI}$ and HA-AKI. Multivariate analysis using binary logistic regression enter method showed that the risk factors for CA-AKI (CA-AKI versus non-AKI) and HA-AKI (HA-AKI versus non-AKI), respectively. To construct the Cox model, univariate Cox regression for each predicting variable (sex, age, preexisting medical conditions, complications, requirement for mechanical ventilation, requirement for RRT, ICU admission, AKI severity, presence of HA-AKI, or CA-AKI) was carried out, with the inpatient mortality of patients with AKI as the outcome variable. Variables that were found to be significant on univariate Cox models were entered into the multivariable Cox model. Kaplan-Meier survival analysis was done to assess inpatient mortality. $P<0.05$ was considered significant.

\section{Results AKI prevalence}

In this study, there were 1,823 adult admissions to hospital, of which $55(3.0 \%)$ cases were excluded. A total of 1,768 patients were enrolled for evaluation. Most (76\%) patients were male. The mean age of the overall cohort was 77.0 years (SD: 9.1). Overall, 377 patients with AKI were identified, giving an overall AKI prevalence of $21.3 \%$. A total of 280 (74.3\%) patients had developed AKI in the community and were admitted to hospital with AKI (CA-AKI). The overall prevalence of CA-AKI was $15.8 \%$ of all hospital admissions. The remaining 97 (25.7\%) AKI episodes were acquired within hospital (HA-AKI). The overall prevalence of HA-AKI was $5.5 \%$ of all hospital admissions.

\section{AKI characteristics}

Thirteen percent of AKI episodes were severe (stage 3 AKI), 62\% had stage 1 AKI, and 25\% had stage 2 AKI. Demographic differences between patients with HA-AKI and CA-AKI are shown in Table 1.

The number of men and women in HA-AKI and CAAKI groups was similar. There was no significant difference in age between HA-AKI and CA-AKI groups (83 years versus 81 years; $P=0.12$ ). Patients with HA-AKI were more likely to have severe AKI $(22 \%$ had stage 3 AKI in the HA-AKI group compared with $11 \%$ in the CA-AKI

Table I Demographic data: CA-AKI versus HA-AKI

\begin{tabular}{|c|c|c|c|}
\hline Variables & $\begin{array}{l}\text { CA-AKI } \\
(n=280)\end{array}$ & $\begin{array}{l}\text { HA-AKI } \\
(\mathrm{n}=97)\end{array}$ & $P$-value \\
\hline Age (years) & $81(77-86)$ & $83(76-87)$ & 0.12 \\
\hline Men, n (\%) & $2 \mid 4(76.4)$ & $76(79.2)$ & 0.58 \\
\hline $\begin{array}{l}\text { Peak serum creatinine } \\
(\mu \mathrm{mol} / \mathrm{L})\end{array}$ & $137(\mid 22-182)$ & 143 (II9-194) & 0.85 \\
\hline Nephrology consultation (\%) & $26(9.3)$ & II (II.5) & 0.54 \\
\hline \multicolumn{4}{|l|}{ AKI severity, n (\%) } \\
\hline Stage I & $175(62.5)$ & $58(59.8)$ & 0.64 \\
\hline Stage 2 & $75(26.8)$ & $18(18.6)$ & 0.11 \\
\hline Stage 3 & $30(10.7)$ & $21(21.6)$ & 0.007 \\
\hline \multicolumn{4}{|l|}{ Complications, n (\%) } \\
\hline Acute respiratory failure & $122(43.6)$ & $44(45.8)$ & 0.70 \\
\hline $\begin{array}{l}\text { Hypercapnic } \\
\text { encephalopathy }\end{array}$ & $24(8.6)$ & $8(8.3)$ & 1.00 \\
\hline \multicolumn{4}{|l|}{ Comorbidity, n (\%) } \\
\hline Chronic cor pulmonale & $117(4 \mid .8)$ & $59(6 \mid .5)$ & 0.001 \\
\hline $\begin{array}{l}\text { Pulmonary arterial } \\
\text { hypertension }\end{array}$ & $9(3.2)$ & $7(7.3)$ & 0.14 \\
\hline Atrial fibrillation & $43(I 5.4)$ & $16(16.7)$ & 0.76 \\
\hline Hypertension & $169(60.4)$ & $56(58.3)$ & 0.73 \\
\hline Diabetes mellitus & $56(20.0)$ & 17 (I7.7) & 0.62 \\
\hline Coronary artery disease & $122(43.6)$ & $38(39.6)$ & 0.50 \\
\hline Chronic kidney disease & $61(21.8)$ & $10(10.4)$ & 0.015 \\
\hline Chronic liver disease & $12(4.3)$ & $4(4.2)$ & 1.00 \\
\hline Anemia & $|2|(43.2)$ & $39(40.6)$ & 0.66 \\
\hline Cerebrovascular disease & $77(27.5)$ & $22(22.9)$ & 0.38 \\
\hline Cancer & $22(7.9)$ & $9(9.4)$ & 0.64 \\
\hline
\end{tabular}

Notes: Continuous, normally distributed data were analyzed using the Student's $t$-test. Non-normally distributed variables were analyzed using the Mann-Whitney U-test. Analyses of the spread of categorical variables were calculated using the Pearson chi-squared test or Fisher's exact test where appropriate. Data were continuous variables and skew distribution, which were presented as median values.

Abbreviations: AKI, acute kidney injury; CA-AKI, community-acquired $\mathrm{AKI}$; HA-AKI, hospital-acquired AKI. 
group; $P=0.007)$. Nevertheless, the peak $\mathrm{SCr}$ value in the HA-AKI group was similar to that in the CA-AKI group. The prevalence of complications (ARF and hypercapnic encephalopathy) between the two groups was also similar. Comparison of the prevalence of various comorbidities in patients with HA-AKI or CA-AKI revealed approximately equal proportions of anemia, hypertension, diabetes mellitus, cancer, and liver, heart, and cerebrovascular diseases. The only two significant differences were a higher prevalence of chronic cor pulmonale in patients with HA-AKI $(61.5 \%$ versus $41.8 \%$, respectively; $P=0.001$ ) and $\mathrm{CKD}$ in patients with CA-AKI (21.8\% versus $10.4 \%$, respectively; $P=0.015$ ). Only $\sim 10.0 \%$ (37/377) of patients with AKI had a nephrology consultation.

\section{Risk factors for CA-AKI and HA-AKI}

Risk factors for CA-AKI and HA-AKI were similar. CA-AKI and HA-AKI shared several independent risk factors, such as being elderly, requirement for mechanical ventilation, and a history of coronary artery disease and CKD. However, there were two additional independent risk factors for HA-AKI: chronic cor pulmonale (odds ratio [OR], 1.98; 95\% confidence interval $[\mathrm{CI}], 1.24-3.25 ; P=0.004)$ and cancer (OR, 2.70; 95\% CI, 1.28-5.72; $P=0.009$ ).

\section{In-hospital outcomes}

Short-term outcomes in patients with CA-AKI and HA-AKI are shown in Figure 2. Patients with HA-AKI were more likely to require non-invasive mechanical ventilation compared with patients with CA-AKI (31.3\% versus $16.8 \% ; P=0.003)$. Moreover, patients with HA-AKI had a longer duration of mechanical ventilation (median, 11 days; interquartile ratio [IQR], 7-17 days) compared with patients with CA-AKI ( 8 days; $4-15$ days) $(P=0.020)$ (Table 2). However, there were no significant differences in the number of patients requiring mechanical ventilation (46.4\% for HA-AKI versus 36.1\% for CA-AKI), invasive mechanical ventilation (15.5\% for HA-AKI versus $19.3 \%$ for CA-AKI), RRT (4.1\% for HA-AKI versus $2.9 \%$ for CA-AKI), and ICU admission (38.1\% for HA-AKI versus $32.1 \%$ for $\mathrm{CA}-\mathrm{AKI}$ ).

\section{Duration of hospitalization}

Patients with HA-AKI had longer hospitalization (median, 14 days; IQR, 9-20 days) compared with patients with CA-AKI (12 days; 8-17 days) $(P=0.038)$. However, there was no significant difference between the duration of ICU stay (median, 11 days; IQR, 6-16 days) in the HA-AKI group compared with patients with CA-AKI (9 days; 6-17 days) $(P=0.863)$ (Table 2).

\section{Inpatient mortality}

All patients were followed up until death in hospital or upon hospital discharge. During this period, the overall inpatient mortality was $5.9 \%$ (105/1768). The overall mortality was $18.0 \%$ in inpatients with AKI compared with $2.7 \%$ for

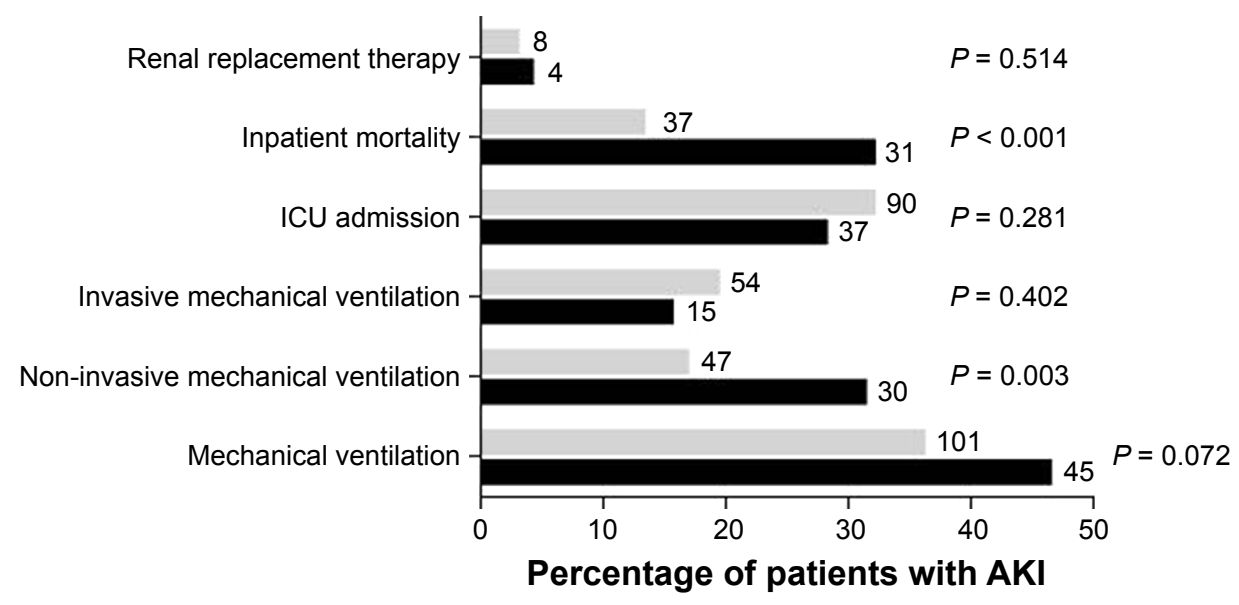

CA-AKI $\longrightarrow$ HA-AKI

Figure 2 In-hospital outcomes for CA-AKI versus HA-AKI.

Notes: This graph demonstrates the percentage of AKI episodes that necessitated renal replacement therapy, ICU admission, mechanical ventilation, invasive mechanical ventilation, and non-invasive mechanical ventilation during hospitalization. This figure shows a comparison of inpatient mortality between CA-AKI and $\mathrm{HA}-\mathrm{AKI}$. The numbers at the top of the individual bars reflect the actual numbers of AKI episodes within this group. $P$-values were calculated using the Pearson chi-squared test/Fisher's exact test where appropriate.

Abbreviations: AKI, acute kidney injury; CA-AKI, community-acquired AKI; HA-AKI, hospital-acquired AKI; ICU, intensive care unit. 
Table 2 Duration of stay in CA-AKI versus HA-AKI

\begin{tabular}{|c|c|c|c|}
\hline Variables & CA-AKI & HA-AKI & $P$-value \\
\hline $\begin{array}{l}\text { Duration of mechanical ventilation } \\
\text { (IQR) (days) }\end{array}$ & $8(4-15)$ & II (7-17) & 0.020 \\
\hline Duration of ICU stay (IQR) (days) & $9(6-17)$ & II (6-16) & 0.863 \\
\hline Duration of hospitalization (IQR) (days) & $12(8-17)$ & $14(9-20)$ & 0.038 \\
\hline
\end{tabular}

Abbreviations: $\mathrm{AKI}$, acute kidney injury; CA-AKI, community-acquired $\mathrm{AKI}$; HA-AKI, hospital-acquired AKI; ICU, intensive care unit; IQR, interquartile ratio.

inpatients without AKI. Moreover, mortality was higher in patients with HA-AKI (32.0\%) than in those with CA-AKI $(13.2 \%)(P<0.001)$. Figure 3 shows the Kaplan-Meier survival curves for CA-AKI and HA-AKI groups. Patients with HA-AKI carried a greater risk for inpatient mortality than those with CA-AKI (OR, 3.09; 95\% CI, 1.78-5.34; $P<0.001$, binary regression analysis). Table 3 demonstrates the independent contribution of listed covariates on the mortality of inpatients with AKI. After adjustment for other significant factors of death, the hazard ratio (HR) for inpatient mortality in the HA-AKI group versus the CA-AKI group was $1.70(95 \% \mathrm{CI}, 1.03-2.81 ; P=0.038)$.

\section{Mortality according to AKI severity}

AKI stage 3 was an independent predictor for inpatient mortality (HR, 1.40; 95\% CI, 1.02-1.92; $P=0.036$ ) (Table 2). Moreover, a significant percentage of patients with stage 3 CA-AKI and HA-AKI had higher mortality $(P<0.001)$. In particular, $67 \%$ of patients with stage 3 HA-AKI and

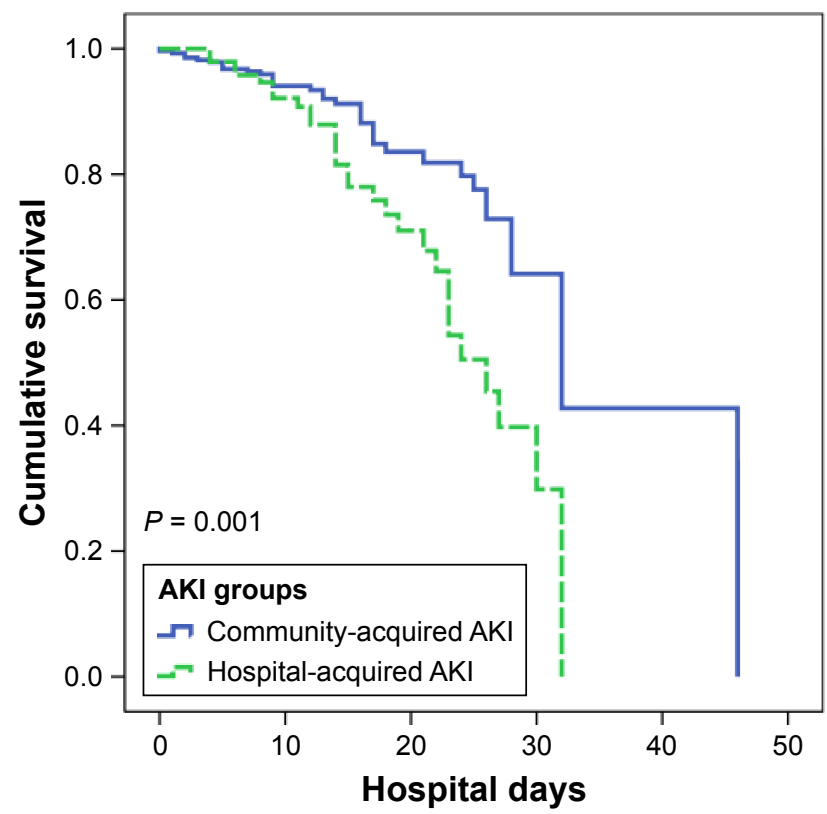

Figure 3 Kaplan-Meier survival curves comparing community-acquired AKI and hospital-acquired AKI groups.

Abbreviation: AKI, acute kidney injury.
Table 3 Cox regression survival analyses for inpatient mortality

\begin{tabular}{|c|c|c|}
\hline Variables & $\begin{array}{l}\text { Hazard ratio } \\
(95 \% \mathrm{Cl})\end{array}$ & $P$-value \\
\hline Admission to ICU & $1.32(0.62-2.79)$ & 0.47 \\
\hline Mechanical ventilation & $0.86(0.45-1.67)$ & 0.67 \\
\hline Acute respiratory failure & $3.19(1.63-6.24)$ & 0.001 \\
\hline Renal replacement therapy & $2.65(1.21-5.78)$ & 0.015 \\
\hline $\begin{array}{l}\text { AKI stage } 3 \text { compared with AKI } \\
\text { stages I and } 2\end{array}$ & $1.40(1.02-1.92)$ & 0.036 \\
\hline HA-AKI compared with CA-AKI & $1.70(1.03-2.8 \mathrm{I})$ & 0.038 \\
\hline
\end{tabular}

Abbreviations: AKI, acute kidney injury; CA-AKI, community-acquired AKI; $\mathrm{Cl}$, confidence interval; HA-AKI, hospital-acquired AKI; ICU, intensive care unit.

$47 \%$ of patients with stage 3 CA-AKI died during hospitalization (Figure 4).

\section{Discussion}

We found that nearly one-fifth of patients with AECOPD requiring hospitalization had evidence of AKI. Of them, three-quarters had CA-AKI, and one-quarter had HA-AKI. CA-AKI and HA-AKI demonstrated similar demographic and risk factors. Nevertheless, patients with HA-AKI had worse short-term outcomes. In comparison with CA-AKI, patients with HA-AKI were more likely to require noninvasive mechanical ventilation and had a longer duration of mechanical ventilation, longer hospitalization, and higher inpatient mortality. After adjustment for other significant factors, HA-AKI (compared with CA-AKI) remained an independent risk factor for the mortality of inpatients with AKI.

\section{Inpatient mortality outcomes according to AKI severity}

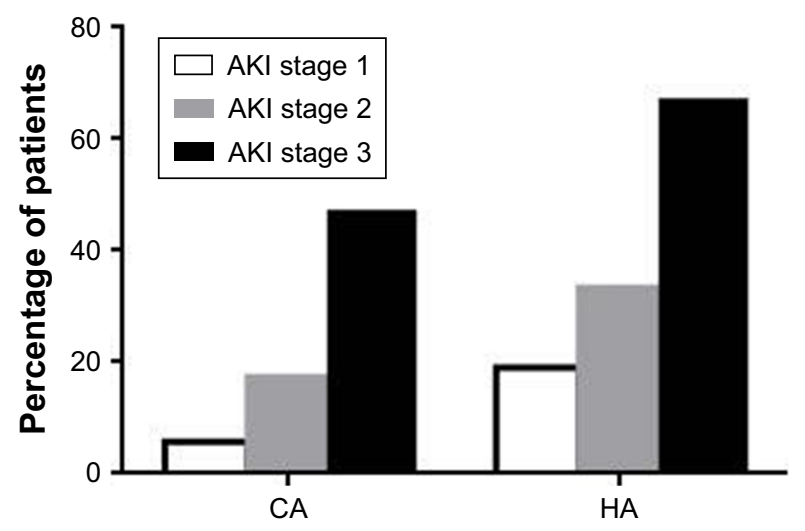

Figure $\mathbf{4}$ Inpatient mortality outcomes according to AKI stage.

Notes: This figure details the percentage of patients in each AKI stage who died during hospitalization. $P<0.00$ I for comparison between inpatient death in AKI stage 3 (Pearson chi-squared test). For AKI stages I and 2, comparisons of inpatient mortality between CA-AKI and HA-AKI groups were not significant.

Abbreviations: $\mathrm{AKI}$, acute kidney injury; CA-AKI, community-acquired $\mathrm{AKI}$; HA-AKI, hospital-acquired AKI. 
We estimated an AKI prevalence of $21.3 \%$ in patients with AECOPD requiring hospitalization. Our estimate was much higher than the prevalence of $1.9 \%$ reported by Barakat and coworkers. ${ }^{5}$ Three explanations may account for this difference. First, in the study by Barakat et al, ${ }^{5}$ AKI was identified through the International Classification of Diseases, which may be presented inaccurately and underestimate the prevalence of AKI. Second, since 2008, the prevalence of AKI coding has been increasing steadily (noted by Kolhe ${ }^{8}$ in a study started in 2004). Hence, the AKI prevalence may have been underestimated. Third, nearly one-third of patients in this study were seriously ill, complicated by ARF. Previous studies have demonstrated that the prevalence of AKI in patients with ARF is $35-62 \% .{ }^{9-11}$ Our data showed that CA-AKI was more common than HA-AKI. The prevalence of CA-AKI was approximately threefold higher than that of HA-AKI (15.8\% versus 5.5\%). This result confirmed the findings from the studies in different practice settings in which the prevalence of CA-AKI was 1.5-4.0-fold higher than that of HA-AKI. ${ }^{12-17}$ In particular, AKI was common in the community for patients with AECOPD requiring hospitalization. Thus, it was necessary to measure $\mathrm{SCr}$ levels and evaluate renal function upon hospital admission for those patients.

This study showed that the risk factors for CA-AKI and HAAKI were similar. CA-AKI and HA-AKI were more common in elderly patients with preexisting coronary artery disease, $\mathrm{CKD}$, and requiring mechanical ventilation. These data demonstrated the demographic characteristics of people in the community and hospital who may benefit from more frequent blood tests in the event of medication change or an acute illness.

Compared with CA-AKI, patients with HA-AKI had worse short-term outcomes. ${ }^{12-17}$ Patients with HA-AKI frequently required more care in the ICU, $,^{13,14,16,17} \mathrm{RRT},{ }^{15}$ and mechanical ventilation ${ }^{12,13}$ and had longer hospitalization ${ }^{14-16}$ and higher inpatient mortality. ${ }^{12,14-17}$ In this study, patients with HA-AKI were more likely to require non-invasive mechanical ventilation and had a longer duration of mechanical ventilation. Higher inpatient mortality was noted in the HA-AKI group. In addition, patients with HA-AKI had longer hospitalization. Non-invasive ventilation is an evidence-based treatment recommended for patients admitted with AECOPD. ${ }^{18,19}$ However, mechanical ventilation was also a common risk factor for AKI in this study. Kuiper et $\mathrm{al}^{20}$ reported that mechanical ventilation may lead to AKI through hemodynamic alterations with reduced renal blood flow or ventilator-induced lung injury by triggering a pulmonary inflammatory reaction and subsequent systemic release of pro-inflammatory mediators. Moreover, several studies have examined the release of these mediators during mechanical ventilation. ${ }^{21-24}$ This may explain (at least in part) why patients developed HA-AKI during hospitalization, and why patients with more severe AKI were in the HA-AKI group. Moreover, our results demonstrated that HA-AKI, severe AKI (stage 3), RRT, and ARF were independent risk factors for inpatient mortality during AKI episodes. In this study, the prevalence of ARF and requirement of RRT in patients with CA-AKI and HA-AKI were similar. However, more patients with severe AKI were in the HA-AKI group, which may explain the higher mortality in inpatients with HA-AKI.

Barakat and coworkers ${ }^{5}$ reported that mortality among patients with AKI at the first COPD exacerbation was $512 / 10,000$ person-years. Our data showed that the overall mortality of inpatients with AKI upon a COPD exacerbation was $18.0 \%$, and the inpatient mortality in the HA-AKI group and the CA-AKI group was $32.0 \%$ and $13.2 \%$, respectively. Importantly, the mortality of inpatients with AKI stage 3 in the HA-AKI group and the CA-AKI group was $67 \%$ and $47 \%$, respectively.

Scholars have reported that a delayed nephrology consultation could be associated with worse outcomes. ${ }^{25,26}$ Ponce et $\mathrm{al}^{26}$ stressed the role of an early nephrology consultation in outcome for AKI patients. The authors concluded that a delayed nephrology consultation was associated with increased mortality among non-dialyzed patients $(53 \%$ versus $22 \%, P<0.01)$ and dialyzed patients ( $74 \%$ versus $49 \%, P<0.006)$ as well as increased duration of stay in the ICU (17 versus 6 days, $P<0.001$ ) and hospital (median, 19 versus 16 days, $P<0.01) .{ }^{25}$ Ponce et al ${ }^{26}$ reported that a delayed nephrology consultation was associated with increased mortality in the ICU $(88.2 \%$ versus $65.4 \%$, $P<0.001)$. However, only $10 \%$ (37/377) of patients with AKI consulted nephrologists in this study. In future, studies with a larger patient cohort could be employed to explore the association between an early nephrology consultation and outcomes in patients with HA-AKI and CA-AKI.

This study had four main limitations. First, it was a retrospective single-center study. A multicenter prospective study with a larger patient cohort is needed to confirm our conclusions. Second, information was not obtained on the differences in patient care that may have influenced the ultimate outcome. Drugs such as diuretics, non-steroidal antiinflammatory drugs, and aminoglycosides are risk factors for AKI. ${ }^{27}$ Third, there was no information on the long-term (eg, mortality, renal) outcomes of AKI. Four, the COPD severity stage is an important characteristic for patients with AECOPD, and we lacked these data. 


\section{Conclusion}

We found that AKI was common in patients with AECOPD requiring hospitalization. $\mathrm{CA}-\mathrm{AKI}$ was more common than HA-AKI but otherwise demonstrated similar demographics and risk factors. Nevertheless, patients with HA-AKI had worse short-term outcomes. Therefore, preventive and management strategies for AKI must be identified, implemented, and evaluated to limit the risks to susceptible individuals.

\section{Acknowledgment}

This study was supported by the Specific Project for Technology Clinical Medicine of Jiangsu Province (BL2014015) and the Key Program for Development of Technology of Nanjing (201405031).

\section{Author contributions}

$\mathrm{XW}$ conceived the study and is the guarantor of the manuscript. C-CC and D-WC prepared the first draft and finalized the manuscript based on comments from all other authors. JL, $\mathrm{M}-\mathrm{QM}$, and Y-BC undertook analyses and interpretation of results. Y-ZC, XH, and WS participated in the preparation and analyses of data and proffered a critique of the manuscript. All authors contributed toward data analysis, drafting and critically revising the paper and agree to be accountable for all aspects of the work. All authors agreed to be accountable for all aspects of the work.

\section{Disclosure}

The authors report no conflicts of interest in this work.

\section{References}

1. World Health Organization [webpage on the Internet]. Chronic Obstructive Pulmonary Disease (COPD). 2014. [cited July 23, 2014]; Available from: http://www.who.int/respiratory/copd/en/. Accessed January 6, 2018.

2. Celli BR, Barnes PJ. Exacerbations of chronic obstructive pulmonary disease. Eur Respir J. 2007;29(6):1224-1238.

3. Wedzicha JA, Seemungal TA. COPD exacerbations: defining their cause and prevention. Lancet. 2007;370(9589):786-796.

4. Fabbian F, De Giorgi A, Manfredini F, et al. Impact of renal dysfunction on in-hospital mortality of patients with severe chronic obstructive pulmonary disease: a single-center Italian study. Int Urol Nephrol. 2016;48(7):1121-1127.

5. Barakat MF, McDonald HI, Collier TJ, Smeeth L, Nitsch D, Quint JK. Acute kidney injury in stable COPD and at exacerbation. Int $J$ Chron Obstruct Pulmon Dis. 2015;10:2067-2077.

6. Vestbo J, Hurd SS, Agusti AG, et al. Global strategy for the diagnosis, management, and prevention of chronic obstructive pulmonary disease: GOLD executive summary. Am J Respir Crit Care Med. 2013;187(4): 347-365.

7. Kellum JA, Lameire N, KDIGO AKI Guideline Work Group. Diagnosis, evaluation, and management of acute kidney injury: a KDIGO summary (Part 1). Crit Care. 2013;17(1):204.

8. Kolhe $\mathrm{N}$ [webpage on the Internet]. Epidemiology of Acute Kidney Injury in England - 1998 to 2013. 2014. [cited April 29, 2015]. Available from: https://clinicaltrials.gov/ct2/show/NCT02216695. NLM identifier: NCT02216695. Accessed January 6, 2018.
9. Liu KD, Glidden DV, Eisner MD, et al. Predictive and pathogenetic value of plasma biomarkers for acute kidney injury in patients with acute lung injury. Crit Care Med. 2007;35(12):2755-2761.

10. Mandelbaum T, Scott DJ, Lee J, et al. Outcome of critically ill patients with acute kidney injury using the Acute Kidney Injury Network criteria. Crit Care Med. 2011;39(12):2659-2664.

11. Soto GJ, Frank AJ, Christiani DC, Gong MN. Body mass index and acute kidney injury in the acute respiratory distress syndrome. Crit Care Med. 2012;40(9):2601-2608.

12. Cui Z, Wang Y, Fan MH. 医院获得性与社区获得性急性肾功能 衰竭临床特点比较 [Comparison of clinical characteristics between hospital-acquired and community-acquired acute renal failure]. Zhongguo Wei Zhong Bing Ji Jiи Yi Xие. 2005;17(10):615-618. Chinese.

13. Der Mesropian PJ, Kalamaras JS, Eisele G, Phelps KR, Asif A, Mathew RO. Long-term outcomes of community-acquired versus hospital-acquired acute kidney injury: a retrospective analysis. Clin Nephrol. 2014;81(3):174-184.

14. Wonnacott A, Meran S, Amphlett B, Talabani B, Phillips A. Epidemiology and outcomes in community-acquired versus hospital-acquired AKI. Clin J Am Soc Nephrol. 2014;9(6):1007-1014.

15. Hsu CN, Lee CT, $\mathrm{Su} \mathrm{CH}$, et al. Incidence, outcomes, and risk factors of community-acquired and hospital-acquired acute kidney injury: a retrospective cohort study. Medicine (Baltimore). 2016;95(19):e3674.

16. Schissler MM, Zaidi S, Kumar H, Deo D, Brier ME, McLeish KR. Characteristics and outcomes in community-acquired versus hospitalacquired acute kidney injury. Nephrology (Carlton). 2013;18(3): $183-187$.

17. Obialo CI, Okonofua EC, Tayade AS, Riley LJ. Epidemiology of de novo acute renal failure in hospitalized African Americans: comparing community-acquired vs hospital-acquired disease. Arch Intern Med. 2000;160(9):1309-1313.

18. National Collaborating Centre for Chronic Conditions. Chronic obstructive pulmonary disease. National clinical guideline on management of chronic obstructive pulmonary disease in adults in primary and secondary care. Thorax. 2004;59(suppl 1):1-232.

19. Ram FS, Picot J, Lightowler J, et al. Non-invasive positive pressure ventilation for treatment of respiratory failure due to exacerbations of chronic obstructive pulmonary disease. Cochrane Database Syst Rev. 2004;(3):CD004104.

20. Kuiper JW, Groeneveld AB, Slutsky AS, Plötz FB. Mechanical ventilation and acute renal failure. Crit Care Med. 2005;33(6):1408-1415.

21. Ranieri VM, Suter PM, Tortorella C, et al. Effect of mechanical ventilation on inflammatory mediators in patients with acute respiratory distress syndrome: a randomized controlled trial. JAMA. 1999;282(1): 54-61.

22. Gurkan OU, O’Donnell C, Brower R, Ruckdeschel E, Becker PM. Differential effects of mechanical ventilatory strategy on lung injury and systemic organ inflammation in mice. Am J Physiol Lung Cell Mol Physiol. 2003;285(3):L710-L718.

23. Imai Y, Parodo J, Kajikawa O, et al. Injurious mechanical ventilation and end-organ epithelial cell apoptosis and organ dysfunction in an experimental model of acute respiratory distress syndrome. JAMA. 2003;289(16):2104-2112.

24. Hepokoski M, Englert JA, Baron RM, et al. Ventilator-induced lung injury increases expression of endothelial inflammatory mediators in the kidney. Am J Physiol Renal Physiol. 2017;312(4):F654-F660.

25. Mehta RL, McDonald B, Gabbai F, et al. Nephrology consultation in acute renal failure: does timing matter? Am J Med. 2002;113(6): 456-461.

26. Ponce D, Zorzenon Cde P, dos Santos NY, Balbi AL. Early nephrology consultation can have an impact on outcome of acute kidney injury patients. Nephrol Dial Transplant. 2011;26(10):3202-3206.

27. Rewa O, Bagshaw SM. Acute kidney injury-epidemiology, outcomes and economics. Nat Rev Nephrol. 2014;10(4):193-207. 
International Journal of COPD

Dovepress

\section{Publish your work in this journal}

The International Journal of COPD is an international, peer-reviewed journal of therapeutics and pharmacology focusing on concise rapid reporting of clinical studies and reviews in COPD. Special focus is given to the pathophysiological processes underlying the disease, intervention programs, patient focused education, and self management protocols.

This journal is indexed on PubMed Central, MedLine and CAS. The manuscript management system is completely online and includes a very quick and fair peer-review system, which is all easy to use. Visit http://www.dovepress.com/testimonials.php to read real quotes from published authors.

Submit your manuscript here: http://www.dovepress.com/international-journal-of-chronic-obstructive-pulmonary-disease-journal 